\title{
ARTICLE
}

\section{Unique formulations in TITAN and PENTRAN for medical physics applications}

\author{
Alireza Haghighat ${ }^{\mathrm{a}^{*}}$, Katherine Royston ${ }^{\mathrm{a}}$, Glenn Sjoden ${ }^{\mathrm{b}}, \mathrm{Ce} \mathrm{Yi}^{\mathrm{b}}$ and Mi Huang ${ }^{\mathrm{b}}$ \\ ${ }^{a}$ Nuclear Engineering Program, Mechanical Engineering Dept, Virginia Tech, Arlington, Virginia, USA; ${ }^{b}$ Nuclear and Radiological \\ Engineering and Medical Physics Program, Georgia Tech, Atlanta, Georgia, USA
}

\begin{abstract}
Special algorithms for application to medical physical problems have been developed and implemented into the TITAN and PENTRAN 3-D parallel transport codes. TITAN is a 3-D parallel transport code with hybrid algorithms including 1) Sn and Characteristics, and 2) Sn with a fictitious quadrature set and ray-tracing. PENTRAN is a 3-D parallel transport code with adaptive differencing Sn formulation, full domain decomposition, and different angular quadrature types (including a characteristic option) with ordinate splitting. In this paper, we will discuss new algorithms developed in TITAN for image reconstruction in Single Photon Emission Computed Tomography (SPECT). It is demonstrated that TITAN projection images are in excellent agreement with the Monte Carlo MCNP5 predictions, while its computation time was up to 2787 times faster than the MCNP5 computation time for a $0.98^{\circ}$ collimator acceptance angle. We will also discuss a novel algorithm developed in PENTRAN for dose calculations in heterogeneous, voxelized phantoms or other geometries. The new algorithm called EDK-Sn, or "Electron Dose Kernel-Discrete Ordinates (EDK-Sn)" yielded a speedup of $\sim 8$ over traditional highly parallel Monte Carlo calculation times, with $<7 \%$ difference in dose among different organs (or smaller given stochastic uncertainties).
\end{abstract}

\section{Keywords: radiation transport; deterministic; Monte Carlo; SPECT imaging; radiation therapy; TITAN; PENTRAN}

\section{Introduction}

Special algorithms for application to medical physical problems have been developed and implemented into the TITAN [1, 2] and PENTRAN (Parallel Environment Neutral-particle TRANsport) [3] 3-D parallel transport codes. TITAN is a 3-D parallel transport code with hybrid algorithms including 1) Sn and Characteristics, [2] and 2) Sn with a fictitious quadrature set and ray-tracing [4]. PENTRAN is a 3-D parallel transport code with an adaptive differencing Sn formulation, full domain decomposition, and different angular quadrature types with ordinate splitting [5].

TITAN has been used for simulation of Computed Tomography (CT) and Single Photon Emission Computed Tomography (SPECT) [6] devices. In this paper, we will discuss new algorithms developed for SPECT. Specifically, we will elaborate on the use of a hybrid formulation which is comprised of the use of the $\mathrm{Sn}$ algorithm in the phantom region, a new fictitious quadrature set [4] for directions starting from the phantom surface and passing through each collimator, and a simplified ray-tracing algorithm for transporting particles along the fictitious directions to the gamma camera. The TITAN projection images are compared with the Monte Carlo MCNP5 [7] predictions for collimators of large and low aspect ratios.

With recent interest in single fraction Stereotactic Body Radiation Therapy, quantification of out-of-field dose assessments is increasingly important. A novel, fast convergence dose computation approach using the parallel PENTRAN code called EDK-Sn, or "Electron Dose Kernel-Discrete Ordinates" [8] has been developed for dose calculations in heterogeneous, voxelized phantoms or other geometries. Pre-computed electron dose kernels generated in advance using full physics Monte Carlo are rapidly projected and integrated over a spatial grid using Sn derived voxelized net currents and fluxes to yield accurate radiotherapy dose calculations. The method has been used for both in-field and out-of-field whole body dose calculations.

This paper is organized as follows. Section 2 discusses the novel hybrid formulations for SPECT implemented in TITAN, and compares its performance with a Monte Carlo calculation. Section 3 discusses the EDK-Sn algorithm implemented in PENTRAN, and compares its performance with a Monte Carlo simulation. Section gives concluding remarks. 


\section{TITAN Novel hybrid formulations for SPECT simulation}

In this section, we will describe a SPECT device, discuss the novel hybrid formulation developed in TITAN for simulation of the SPECT, and compare the accuracy and performance of the new formulation with the Monte Carlo MCNP5 predictions.

\subsection{Description of SPECT}

SPECT is a functional imaging modality in which a patient is injected with a radionuclide that is preferentially absorbed by a tissue of interest. The gamma-rays emitted by the radionuclide are then detected at a gamma camera at different angles around the patient to form projection images as depicted in Figure 1.

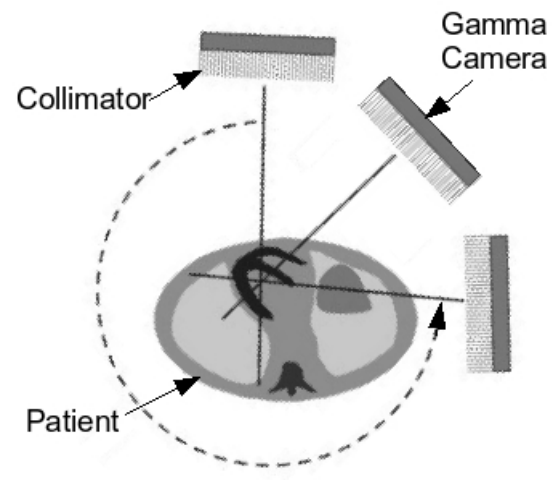

Figure 1. Physical phantom being imaged by a prototype SPECT device.

For this work, a SPECT myocardial perfusion study with Technecium-99m (Tc-99m) was simulated. Tc-99m is absorbed by the heart wall where it emits $140.5 \mathrm{keV}$ gamma rays. The NURBS-based cardiac-torso (NCAT) code [9] was used to create a $64 \times 64 \times 64$ voxel phantom $\left(40 \times 40 \times 40 \mathrm{~cm}^{3}\right)$ with a Tc-99m source in the heart wall. This voxel phantom is used in both the TITAN and MCNP5 simulations.

\subsection{Novel hybrid Sn with fictitious quadrature and ray-tracing in TITAN}

The hybrid formulation in TITAN is comprised of the following steps:

- $\quad$ Step 1 - Sn transport calculation with a regular quadrature set in the phantom region.

- $\quad$ Step 2 - Generation of fictitious quadrature set with circular ordinate splitting for a projection angle.

- $\quad$ Step 3 - One extra transport sweep with the fictitious quadrature set in the phantom region using the converged flux moments in Step 1 to evaluate the scattering source for directions in the fictitious quadrature set.

- $\quad$ Step 4 - Simulation of the projections images with the fictitious quadrature set using the simplified ray-tracing formulation in the collimator region.
Note that Steps $2-4$ are repeated for each projection angle. Step 1 is only required to perform once. For further detail on the above steps, readers should consult Ref. 4.

\subsection{Model descriptions}

In this section, the MCNP5 and TITAN models used for comparison are described.

\subsubsection{MCNP5 Monte Carlo Model}

The geometry of the MCNP5 simulation can be seen in Figure 2. The collimator is modeled in front of the detector as a $5.7 \mathrm{~cm}$ deep block of lead filled with parallel holes. Note that the detector is not actually modeled, but the flux is tallied in that region to create the projection images. The MCNP5 simulation is run until the $1-\sigma$ uncertainty is $\leq 3 \%$ in the heart flux tallies.

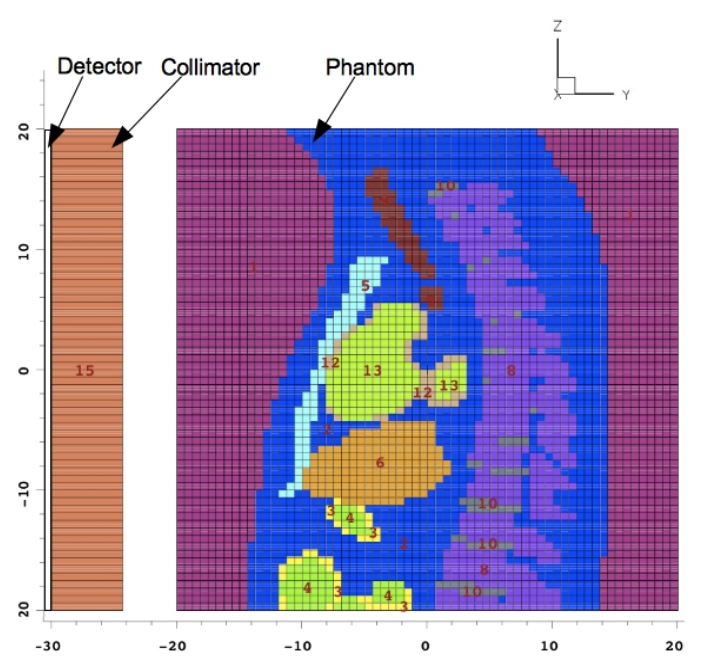

Figure 2. Sagittal slice through MCNP5 model showing NCAT voxel phantom, detector, and collimator positions.

The CEPXS code [10] was used to generate 3-group multi-group cross sections for use in the MCNP5 code. The three energy groups are 126.45-154.55 keV, 98.35-126.45 keV, and 10-98.35 keV. The first group contains the source and was chosen to match the $20 \%$ energy window typically used in SPECT.

\subsubsection{TITAN model}

The TITAN model uses the same 3-group cross sections described in Section 2.3.1. Figure 3 shows that, in the TITAN code's hybrid setting, the Sn solver is used to solve for the flux distribution in the phantom, the Sn solver is used to determine fluxes along fictitious directions on the surface of the phantom, and the simplified ray-tracing algorithm is used for simulating the collimator and calculating the flux at the surface of the detector. 


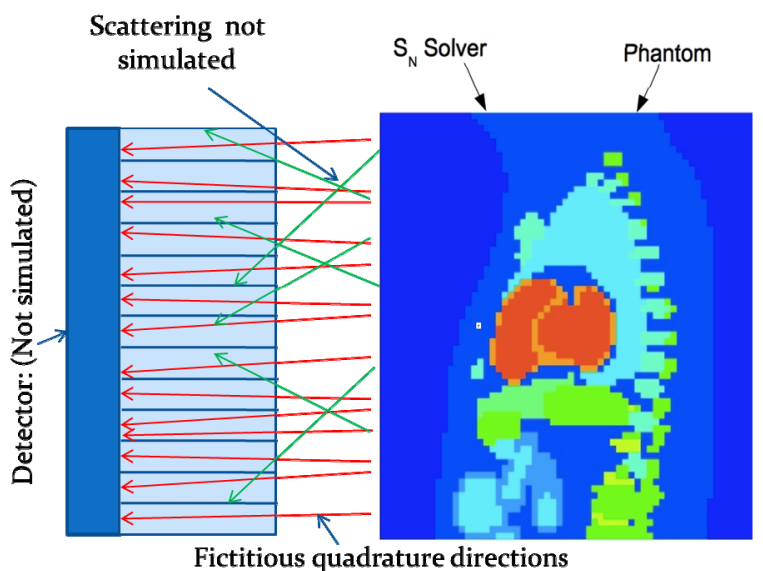

Figure 3. Sagittal slice through TITAN model depicting Sn solver and ray-tracing regions.

\subsection{Results and analysis}

In this section, we will compare projection images generated by TITAN and MCNP5 with each other. As discussed previously, the MCNP5 Monte Carlo simulation was run until the $1-\sigma$ uncertainty was less than $3 \%$ for all of the heart wall pixels. The convergence criterion for the TITAN deterministic simulation was $1 \times 10^{-3}$. Only the high-count pixels in the myocardium are numerically compared between images. The group 1 projection images generated by the TITAN and MCNP5 codes are given in Figure 4. Both figures have been normalized to the highest pixel value.

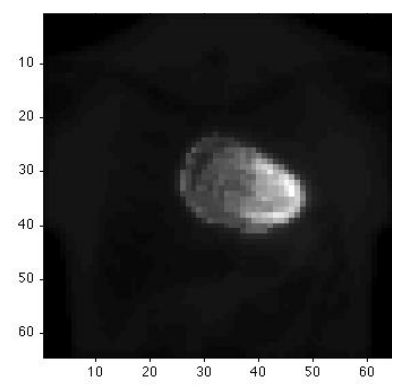

(a)

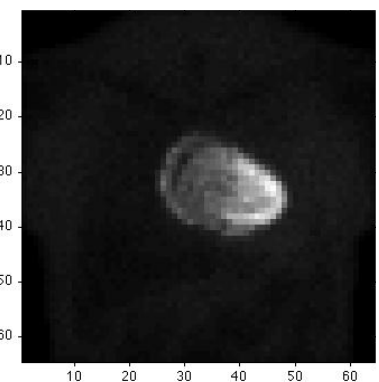

(b)
Figure 4. Anterior projection images for (a) TITAN and (b) MCNP5.

The images in Figure 4 agree well visually. To further test the TITAN code, three different collimator acceptance angles are simulated. The maximum relative difference between the high count heart wall pixels in the MCNP5 and TITAN projection images is given in Table 1 for each collimator case. The projection images in Figure 4 correspond to Case 3.

The results in Table 1 indicate that the differences between TITAN and MCNP5 are reduced as smaller acceptance angles, i.e., small aspect ratio, are modeled. To better understand the differences between the images, profiles through column 44 of the case 3 TITAN and MCNP5 images are given in Figure 5.
Table 1. Maximum difference of TITAN results relative to MCNP5 results for each collimator case

\begin{tabular}{ccc}
\hline $\begin{array}{c}\text { Case } \\
\text { Number }\end{array}$ & $\begin{array}{c}\text { Acceptance Angle } \\
\text { (degrees) }\end{array}$ & $\begin{array}{c}\text { Maximum Relative } \\
\text { Difference (\%) }\end{array}$ \\
\hline 1 & 2.97 & 21.3 \\
2 & 1.42 & 11.9 \\
3 & 0.98 & 8.3 \\
\hline
\end{tabular}

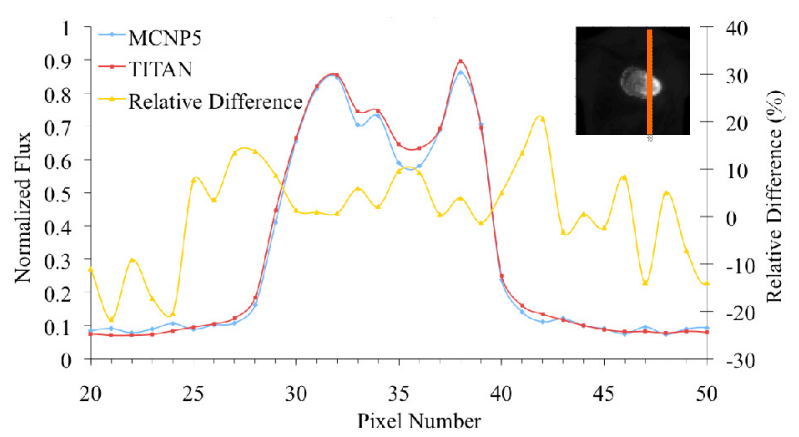

Figure 5. Profiles through column 44 of projection images from TITAN and MCNP5 simulations.

Figure 5 clearly shows peaks in the flux from the heart wall as the profiles pass into and back out of the heart. The profile shapes match well with some amplitude differences in the heart. These differences are attributed to the different methods of representing the collimator used in MCNP5 and TITAN. Note that the TITAN code's ray-tracing with fictitious quadrature technique does not allow for photons to be scattered in the collimator or transmitted through the collimator hole walls.

Parallel timing analysis was also performed and observed a high parallelizable fraction for the TITAN code, in the range of $91-96 \%$. Further results can be found in reference [11]. Timing comparisons with MCNP5 are given in Table 2.

Table 2. Computation time comparison with MCNP5 and TITAN on 16 processors.

\begin{tabular}{|c|c|c|c|c|}
\hline \multirow{2}{*}{$\begin{array}{l}\text { Case } \\
\text { Number }\end{array}$} & \multirow{2}{*}{$\begin{array}{c}\text { Acceptance } \\
\text { Angle } \\
\text { (degrees) }\end{array}$} & \multicolumn{2}{|c|}{ Code } & \multirow{2}{*}{$\begin{array}{c}\text { Speedup Factor } \\
\text { (MCNP5/ } \\
\text { TITAN) }\end{array}$} \\
\hline & & $\begin{array}{l}\text { MCNP5 } \\
\text { (min)* }\end{array}$ & $\begin{array}{l}\text { TITAN } \\
(\min )^{\dagger}\end{array}$ & \\
\hline 1 & 2.97 & 313.8 & 0.82 & 382 \\
\hline 2 & 1.42 & 1071.8 & 0.82 & 1304 \\
\hline 3 & 0.98 & 2289.7 & 0.82 & 2787 \\
\hline
\end{tabular}

*Time to achieve $1-\sigma$ uncertainty of $\leq 3.0 \%$ in the heart ${ }^{\dagger} 180$ projection angles

As demonstrated in Table 2, the TITAN methodology has the advantage of being independent of collimator acceptance angle. The TITAN code is also efficient in that once it has solved for the flux in the phantom, it can quickly calculate projection images without needing to solve the Sn method again. 


\section{EDK-Sn formulation in PENTRAN}

A new methodology called EDK-Sn, or "Electron Dose Kernel-Discrete Ordinates" was developed to accurately estimate organ doses voxelized in the human body principally for applications in high energy external photon beam therapy, accounting for both in-field and out-of-field doses.

The initial computational approach was proposed by G. Sjoden, A. Al-Basheer, M. Ghita in 2008 [8]. This work used a simple, hypothetical flat MV source that was later improved and expanded by Huang and Sjoden [12, 13]. The initial work was tested on an $8 \mathrm{MV}$ flat photon beam source directed at a 15 year old male phantom (middle anterior), reaching agreement with Monte Carlo results in a homogenous phantom to within $10 \%$ in far out-of-field regions, except in strongly heterogeneous regions, where it became difficult to validate the accuracy of the Monte Carlo, compounded by the often significant inherent uncertainties in the stochastic photon-electron simulations [8]. It has been shown that the EDK-Sn method can be an order of magnitude faster than Monte Carlo simulations for whole body dose characterization $[8,12,13]$.

The EDK-Sn methodology was demonstrated using CT-based voxelized anatomical patient phantoms and calibration phantoms. To begin the EDK-Sn procedure, one rapidly solves for the photon transport deterministically over the entire phase space of the phantom using 3-D discrete ordinates (Sn) radiation transport on parallel computer architectures. The photon transport is achieved using the PENTRAN code. Discretization of a phantom is performed as required among the energy, angle, and spatial variables using parallel computation for rapid global solution. The transport photon net current in each Cartesian voxel is derived from the solution of the Boltzmann equation. At the end of this step, the photon flux, photon current, and net current vector direction are determined spanning the phase space of the phantom or CT dataset, as appropriate. Then, this highly detailed angular $\mathrm{Sn}$ data, rendered globally, is used to project the dose and map it to surrounding voxels; the dose is then accumulated by summing on a mesh by mesh basis, scaled by the magnitude of the photon fluence, using Electron Dose Kernels (EDKs).

The EDK-Sn methodology serves as a critical link in a system to accumulate the correct absorbed dose in each fine mesh. Accumulation of the dose in each voxel of tissue is performed, since the accumulated energy deposited in each voxel for each photon energy group is based on the Sn computed photon flux, and is then projected due to electrons streaming along the photon current vector derived from the Sn simulation, as described.

EDK-Sn dose calculations were performed for several slab phantoms using material specific absorbed dose kernels with $1 \mathrm{~cm}$ mesh densities. The dose rate in a soft-tissue phantom, comparing EDK-Sn and Monte Carlo results, is shown in Figure 6; agreement was excellent, and the average relative difference in dose was within a $(2 \sigma)$ statistical Monte Carlo uncertainty.

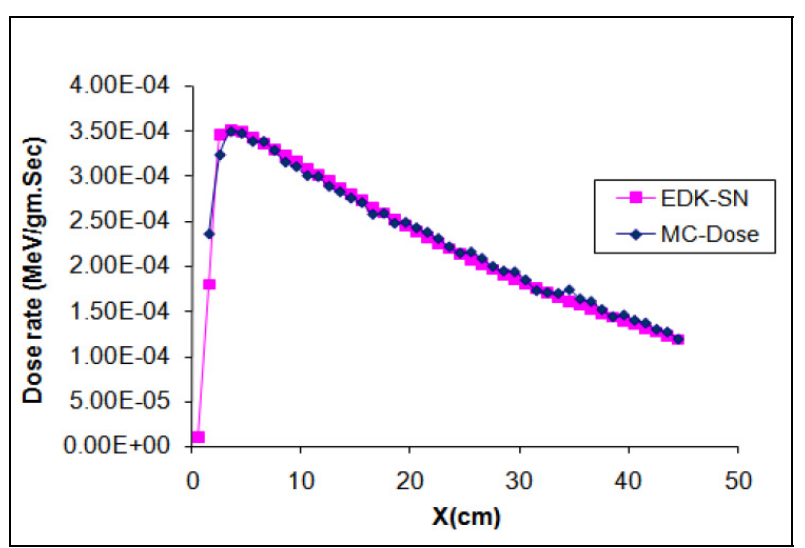

Figure 6. Comparison of EDK-SN dose with on-axis Monte Carlo dose (MCNP *F8 tally) for a tissue slab phantom (MCNP uncertainty $(2 \sigma)$ average $(6.0 \%)$, Average absolute relative difference $(3.7 \%))$.

The EDK-Sn methodology, with density corrections for material heterogeneities, was then applied to a fully voxelized phantom and compared with MCNP5 results for a high energy volumetric $\left(20 \times 1 \times 17 \mathrm{~cm}^{3}\right)$ flat weighted source $[0,8 \mathrm{MeV}]$ using $0.5 \mathrm{MeV}$ binned groups for a total of 16 groups spanning up to $8 \mathrm{MeV}$. The phantom, initially $2 \times 2 \times 2 \mathrm{~mm}^{3}(302 \times 139 \times 836$ voxels), was down sampled to $1 \times 1 \times 1 \mathrm{~cm}^{3}(60 \times 27 \times 167$ voxels), for total of 270,540 voxels; this is shown in Figure 7. Dose Results are indicated in Table 3, which reveal that all doses were comparable within a Monte Carlo $(2 \sigma)$ uncertainty, except for the spleen and prostate. To properly attribute dose due to material heterogeneities in applying the EDK-Sn method, a density scaling correction factor was required to yield good agreement with Monte Carlo results. Monte Carlo prostate doses (using 16 Intel Harpertown cores for 16 hours) were not converged, since this organ was far out of field; subsequent simulations requiring an additional $\sim 40 \mathrm{~h}$ on 16 Intel Harpertown processors demonstrated the Monte-Carlo result was converging to the EDK-Sn result. This demonstrates the power of the EDK-Sn method, since it required a total of $2 \mathrm{~h}(1.5 \mathrm{~h}$ parallel $\mathrm{Sn}$ / $0.5 \mathrm{~h}$ parallel EDK) to yield whole body doses, approximately 8 times faster than the initial Monte Carlo calculation.

\section{Conclusions}

Comparison of the TITAN anterior projection image with the MCNP5 projection showed good visual agreement. For a collimator with a high aspect ratio, the maximum relative difference between the TITAN result and the MCNP5 result was $8.3 \%$. Profiles through the TITAN and MCNP5 projection images matched in shape well with some magnitude differences. These can be attributed to differences in collimator representation. Computation times are far shorter in TITAN with 


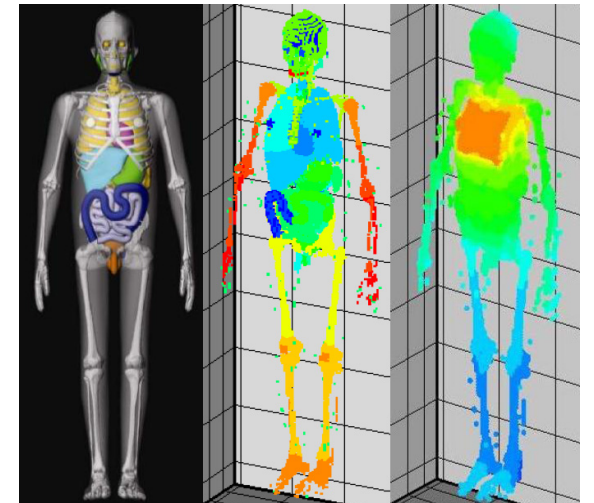

Figure 7. Simulation methodology for EDK-Sn computations using PENTRAN-MP code system. Left: UF Anthropomorphic phantom; (middle) corresponding PENTRAN input, (right): EDK-Sn absorbed dose distribution after application of a $[0,8 \mathrm{MeV}]$ source

Table 3. Comparison of selected organ absorbed dose rate (MeV/g.s) calculated using MCNP5 pulse height tally with (photon, electron mode) and EDK-Sn for the UF hybrid 15 -year-old male phantoms using a $[0,8 \mathrm{MeV}]$ flat weighted source

\begin{tabular}{lcccc}
\hline \multicolumn{1}{c}{ 0rgan } & $\begin{array}{c}\text { MC( }{ }^{*} \text { F8) } \\
\text { (MeV/g.Sec) }\end{array}$ & $\begin{array}{c}\text { (2-sigma) MC } \\
\text { Uncertainty }\end{array}$ & $\begin{array}{c}\text { Sn-EDK } \\
(\text { MeV/g.Sec) }\end{array}$ & $\begin{array}{c}\text { (MC- } \\
\text { EDK)/EDK }\end{array}$ \\
\hline Right+ Left Lung & $1.35 \mathrm{E}-01$ & $6.80 \%$ & $1.41 \mathrm{E}-01$ & $4.56 \%$ \\
Pancreas & $9.47 \mathrm{E}-05$ & $4.14 \%$ & $9.85 \mathrm{E}-05$ & $4.02 \%$ \\
SI W & $3.56 \mathrm{E}-05$ & $4.00 \%$ & $3.75 \mathrm{E}-05$ & $5.43 \%$ \\
Spleen & $1.11 \mathrm{E}-04$ & $3.00 \%$ & $1.18 \mathrm{E}-04$ & $6.58 \%$ \\
Stomach W & $1.86 \mathrm{E}-04$ & $4.60 \%$ & $1.94 \mathrm{E}-04$ & $4.09 \%$ \\
Thyroid & $1.41 \mathrm{E}-05$ & $6.82 \%$ & $1.38 \mathrm{E}-05$ & $2.08 \%$ \\
Prostate & $2.21 \mathrm{E}-08$ & $44.00 \%$ & $2.29 \mathrm{E}-08$ & $3.62 \%$ \\
\hline
\end{tabular}

speedup factors of 382 to 2787 seen for this model. In addition, we presented the EDK-Sn method to rapidly compute doses in phantoms irradiated by high energy external photon beams. Use of the pre-computed EDKs allows for the full charged particle physics dose to be accumulated throughout the voxelized phantom based on a rapidly computed $\mathrm{Sn}$ solution from the PENTRAN code system. To properly attribute dose due to material heterogeneities in applying the EDK-Sn method, a density scaling correction factor was required to yield good agreement with Monte Carlo results. In a fully voxelized phantom problem, all doses were in agreement with those determined by independent Monte Carlo computations when the Monte Carlo results were well converged.

\section{References}

[1] C. Yi, Hybrid Discrete Ordinates and Characteristics Method for Solving the Linear Boltzmann Equation, PhD Thesis, University of
Florida, (2007).

[2] C. Yi and A. Haghighat, A 3-D block-oriented hybrid discrete ordinates and characteristics method, Nuclear Science and Engineering, 164 (3) (2010), pp.221-247.

[3] G. Sjoden and A. Haghighat, PENTRAN: A 3-D cartesian parallel Sn code with angular, energy, and spatial decomposition, Proc. Joint Int'l Conf. on Mathematics and Supercomputing for Nuclear Apps, Saratoga Springs, New York, Vol I (Oct 1997), pp.553-562.

[4] C. Yi and A. Haghighat, Hybrid Sn and ray-tracing with fictitious quadrature for simulation of SPECT, Proc. M\&C 2009, Saratoga Springs, NY, May 3-7, 2009 (2009). [CD-ROM]

[5] G. Longoni and A. Haghighat, Development of the regional angular refinement and its application to the CT-scan device, Trans. Am. Nucl. Soc. 86 (2002), p. 246.

[6] E. G. DePuey, E. V. Garcia and D. S. Berman, Cardiac SPECT Imaging, Lippincott Williams \& Wilkins, Philadelphia, (2001).

[7] X-5 Monte Carlo Team, MCNP-A General Monte Carlo N-Particle Transport Code, Version 5, Los Alamos National Library, (2005).

[8] A. Al-Basheer, G. Sjoden and M. Ghita, Electron dose kernels to account for secondary particle transport in deterministic simulations, Nuclear Technology Journal 168 (2009), pp. 906-918.

[9] W. P. Segars, Development and Application of the New Dynamic NURBS-Based Cardia-Torso (NCAT) Phantom, PhD Thesis, University of North Carolina, (2001).

[10]L. J. Lorence, J. E. Morel and G. D. Valdez, User's Guide to CEPXS/ONELD: A One-Dimensional Coupled Electron-Photon Discrete Ordinates Code Package, Sandia National Laboratory, (1989).

[11]K. Royston, A. Haghighat and C. Yi, Comparison of TITAN hybrid deterministic transport code and MCNP5 for simulation of SPECT, Progress in Nuclear Science and Technology 2, 201 (2011), pp. 201-206.

[12]M. Huang, G. Sjoden, A. Al-Basheer, M. Ghita and W. Bolch, Optimization of electron dose kernels to account for heterogeneities in voxelized phantoms, Proc. IEEE Conference, Orlando, FL, (2009).

[13]M. Huang, G. Sjoden, J. G. Li, A. Al-Basheer and W. Bolch, Validation of a novel dose calculation approach for heterogeneous voxelized phantoms in a parallel computation environment using electron dose kernels for radiotherapy, Proc. AAPM Conference, (2010). 